\title{
EVALUACIÓN POR DURABILIDAD EN MATRICES DE MORTERO BASE CEMENTO PÓRTLAND CON SUSTITUCIÓN DE RESIDUOS GEOTÉRMICOS
}

\author{
V. BÁEZ ${ }^{1}$, E. ALONSO ${ }^{1}$, S. ARGUELLO ${ }^{1}$, C. BERNABÉ ${ }^{1,2}$, M. MONDRAGON $^{1}$, \\ V. BLANCAS ${ }^{1,3}$ \\ ${ }^{1}$ Universidad Michoacana de San Nicolás de Hidalgo, Facultad de Ingeniería Civil, Avenida Francisco. J. Mújica, sin \\ número, Ciudad Universitaria, C.P. 58030, Morelia, Michoacán, México. \\ ${ }^{2}$ Universidad Michoacana de San Nicolás de Hidalgo, Facultad de Ingeniería Química, Avenida Francisco. J. Mújica, sin \\ número, Ciudad Universitaria, C.P. 58030, Morelia, Michoacán, México. \\ ${ }^{3}$ Universidad Michoacana de San Nicolás de Hidalgo, Facultad de Ingeniería Civil, Maestría en Infraestructura del \\ Transporte en la rama de las Vías Terrestres, Avenida Francisco. J. Mújica, sin número, Ciudad Universitaria, C.P. \\ 58030, Morelia, Michoacán, México.
}

\section{RESUMEN}

Se propone utilizar sílice geotérmica como material sustituto puzolanico cementante en la elaboración de morteros con el objetivo de disminuir el consumo de cemento portland (CP) y por ende las emisiones de $\mathrm{CO} 2$ generadas por la industria de la producción del cemento. Se evaluaron las propiedades DURABLES (pérdida de masa por acción de sulfato de sodio) y FÍSICO-MECÁNICAS (compresión simple, velocidad de pulso ultrasónico y resistividad eléctrica) de muestras cubicas de $5 \mathrm{~cm}$, una mezcla control y dos con sustituciones del 5\% de la masa del cemento por sílice. Se observa que estas sustituciones podrían ser un remplazo que disminuye la cantidad de CP utilizado en la elaboración de morteros, proporcionando durabilidad y desempeño mecánico superior que los morteros convencionales.

Palabras clave: Sílice; mortero; geotermia; sustitución; puzolana.

\begin{abstract}
It is proposed to use geothermal silica as a cementing pozzolanic substitute material in the preparation of mortars in order to reduce the consumption of Portland cement $(\mathrm{PC})$ and therefore the $\mathrm{CO} 2$ emissions generated by the cement production industry. The DURABLE properties (loss of mass per action of sodium sulfate) and PHYSICAL-MECHANICAL (simple compression, ultrasonic pulse rate and electrical resistivity) of $5 \mathrm{~cm}$ cubic samples, a control mixture and two with 5\% substitutions of the cement mass per silica. It is noted that these substitutions could be a replacement that decreases the amount of PC used in mortar processing, providing durability and superior mechanical performance than conventional mortars.
\end{abstract}

Keywords: Silica; mortar; geotermal; substitution; pozzolana. 


\section{INTRODUCCIÓN}

El cemento portland es uno de los materiales más empleados en la industria de la construcción, debido fundamentalmente, a las excelentes propiedades mecánicas que presenta en estado endurecido. En México para el año 2016 la producción nacional de cemento Portland alcanzó la cifra de 40.6 millones de toneladas, correspondientes a $324 \mathrm{~kg} / \mathrm{habitante}$ (CANACEM, 2016), cifra que se incrementa como consecuencia del crecimiento poblacional y con ello el desarrollo económico y social. Sin embargo, la producción del $\mathrm{CP}$ es una de las prácticas más contaminantes en el proceso de obtención del clínker, donde la producción de una tonelada de cemento libera cerca de una tonelada de $\mathrm{CO}_{2}$ y gases de $\mathrm{NO}_{2}$ y $\mathrm{SO}_{2}$, asociados a la descarbonatación de la caliza en el horno de clinkerización, así como a la quema de combustibles fósiles.

La energía geotérmica es una de las pocas fuentes de energía renovables capaz de producir electricidad de manera constante las 24 horas del día. El potencial de generación de energía geotérmica a nivel mundial es de entre $70 \mathrm{GW}$ y $80 \mathrm{GW}$. Sin embargo, solo el $15 \%$ de las reservas geotérmicas conocidas en todo el mundo se explota para la producción eléctrica, lo que genera solo $13 \mathrm{GW}$. México tiene actualmente una potencia neta instalada de 720 MW en Cerro Prieto, 193 MW en Los Azufres, 30 MW en Los Humeros y 10 MW en Tres Vírgenes, convirtiéndose así en el cuarto líder mundial como productor de energía geotérmica.

La sílice es la principal incrustación en los campos geotérmicos y se presenta como especie disuelta en todos los líquidos dominantes de los recursos geotérmicos. Los fluidos geotérmicos ascendentes disuelven $\mathrm{SiO} 2$ (sílice amorfa) de las formaciones rocosas y alcanzan la saturación con respecto al cuarzo en sistemas acuosos a altas temperaturas.

Esta investigación está enfocada al tema de los residuos geotérmicos (sílice) utilizados como materiales alternativos en la industria de la construcción. Se presenta la evaluación realizada a mezclas de mortero con sustituciones parciales del $5 \%$ de la masa del cemento por sílice geotérmica. La primera mezcla diseñada con sílice proveniente de la planta geotérmica de Cerro Prieto (Sil CP) ubicada en Baja California, México y la segunda con sílice proveniente de la planta geotérmica los Azufres (Sil Az) ubicada en Michoacán, México.

\section{PROCEDIMIENTO}

El procedimiento empleado para el desarrollo de esta investigación consta básicamente de cuatro fases en las cuales se presenta el diseño del experimento, la preparación de los materiales seleccionados para su ejecución, la elaboración de especímenes, los métodos de prueba y normas empleados.

\subsection{Caracterización de los materiales.}

Para la elaboración de las mezclas de mortero se utilizaron los siguientes materiales: Cemento Portland CPC 30R RS, agua potable para el mezclado proveniente del laboratorio de materiales "Ing. Luis Silva Ruelas" de la Facultad de Ingeniería Civil de la Universidad Michoacana de San Nicolás de Hidalgo, como agregado fino se utilizó una arena volcánica negra proveniente del banco de materiales llamado Joyitas ubicado en la carretera Morelia - Jiquilpan en el kilómetro 14.5 en Michoacán. Se utilizó un aditivo súper plastificante llamado CRISOTAN R-5. El agregado pétreo se caracterizó de acuerdo con la Norma NMX-C-111-ONNCCE-2004. Las muestras se diseñaron con la Norma ASTM-C-109, con una relación agua/cementante de 0.65 y una fluidez $110 \pm 5 \%$.

Para el empleo de la sílice geotérmica en la elaboración de las mezclas de mortero fue necesario llevar a cabo un tratamiento previo de preparación y caracterización, descrito a continuación: 


\subsubsection{Secado de la Sílice geotérmica.}

Con el secado se busca eliminar la humedad presente en el material mediante dos tipos de secado: el primero se realizó al sol por un período de entre 6 y 8 horas continuas (Figura \#1), el segundo se llevó a cabo en hornos a temperatura de $60^{\circ} \mathrm{C}$ por un período de 48 horas.

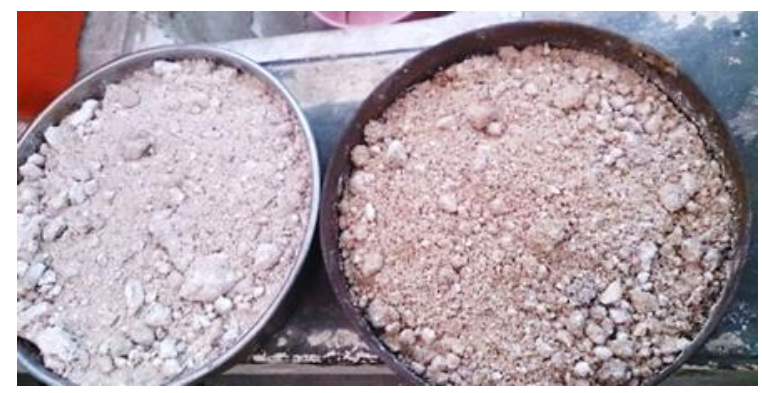

Figura 1. Secado de la sílice al Sol.

2.1.2 Tratamiento mecánico de la Silice Geotérmica: el porcentaje utilizado como sustitución al cemento fue $5 \%$. Se utilizó después de haber sido sometida a un tratamiento mecánico en el molino de desgaste de los ángeles (Figura \#2). Esta decisión se tomó después de haber realizado un cribado del material, en el cual nos dimos cuenta de que al dejar las partículas de mayor tamaño fuera, desperdiciaríamos el $80 \%$ del material, es por esta razón que se decidió moler el material para obtener una mejor reacción con el cemento. Además de que al molerlo aumentamos el área de reacción de las partículas ASTM (C618-15).

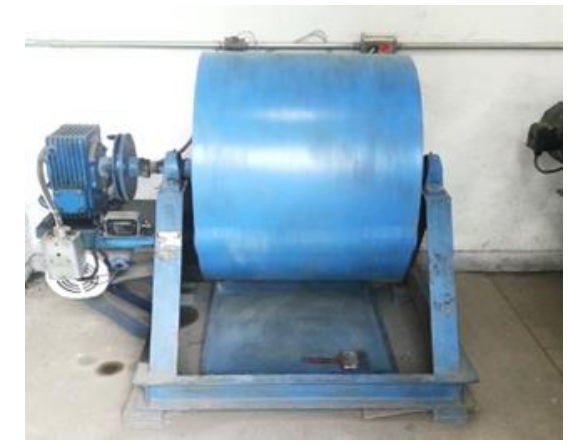

Figura 2. Máquina de desgaste de Los Ángeles.

\subsection{Pruebas realizadas al mortero fresco.}

Para determinar el contenido óptimo de agua en los morteros, las mezclas se diseñaron con una fluidez de $110 \pm 5 \%$. Se diseñó la mezcla por fluidez con base en la norma NMX-C-061-ONNCCE-2015. El objetivo es determinar el contenido de agua necesaria para que el mortero sea manejable, plástico y dúctil. La relación a/c resulto alta en comparación con otras investigaciones debido a que el agregado fino contenía exceso de finos. Ambas mezclas se diseñaron con la misma relación a/c de 0.65.

\subsection{Elaboración de especímenes.}

Una vez que se cumplió con la fluidez, se procedió a elaborar especímenes cúbicos de $5 \mathrm{~cm}$ de arista de acuerdo con la norma ASTM-C-109. El curado de estos se llevó a cabo por inmersión en agua de acuerdo a la norma NMX-C-159-ONNCCE, 2004.

\subsection{Pruebas realizadas al mortero endurecido.}

Las pruebas no destructivas, destructivas y por durabilidad realizadas a lo largo de esta investigación se realizaron bajo las especificaciones de la RED DURAR, normativas NMX y ASTM, bajo las 
condiciones del laboratorio de Materiales “Ing. Luis Silva Ruelas”. Cada resultado de las pruebas realizadas se obtuvo del promedio de 5 especímenes probados por mezcla para cada edad.

\subsubsection{Pruebas No destructivas.}

Velocidad de pulso ultrasónico (V.P.U.) en base a la norma NMX-C-275-ONNCCE-2004 y resistividad eléctrica en base a la norma NMX-C-514-ONNCCE-2016).

\subsubsection{Pruebas Destructivas.}

Compresión simple en base a la norma NMX-C-083- ONNCCE-2014.

\subsubsection{Pruebas por Durabilidad}

Pérdida de masa por accion de sulfato de sodio en base a la norma ASTM-C-88-9a.

\section{RESULTADOS}

De acuerdo con los criterios de evaluación de la Red DURAR para Velocidad de Pulso Ultrasónico (Tabla 1), en la figura \#3 se puede observar que las sustituciones con sílice presentan calidad DURABLE a la edad de 90 días, superando a la mezcla testigo.

Cabe mencionar que los criterios de evaluación de la Red Durar son para concreto, sin embargo, se utilizan en morteros como referencia de calidad.

Tabla 1. Criterio de evaluación de la calidad del concreto hidráulico en función de la VPU (CYTEDDURAR, 1998).

\begin{tabular}{|c|c|c|}
\hline \multirow{2}{*}{$\begin{array}{c}\text { Calidad del } \\
\text { concreto }\end{array}$} & \multicolumn{2}{|c|}{ Velocidad de propagación $(\mathbf{m} / \mathbf{s})$} \\
\cline { 2 - 3 } & Desde & Hasta \\
\hline Deficiente & $<2000$ & \\
\hline Normal & 2001 & 3000 \\
\hline Alta & 3001 & 4000 \\
\hline Durable & $>4000$ & \\
\hline
\end{tabular}

\section{VELOCIDAD DE PULSO ULTRASÓNICO}

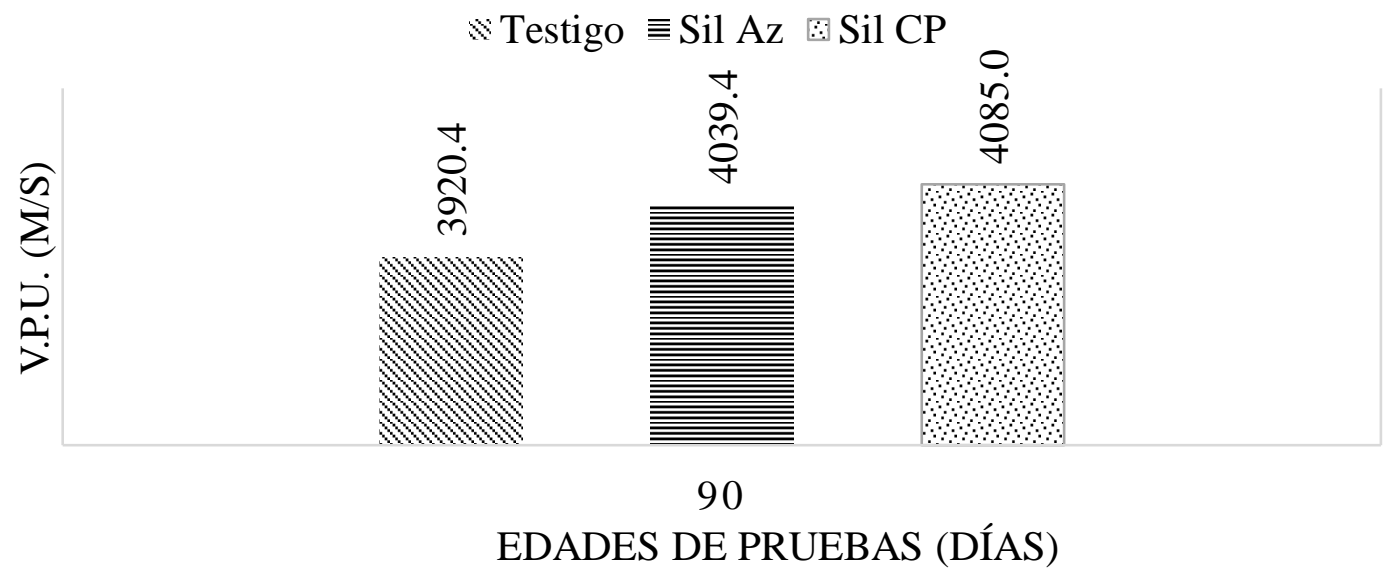

Figura 3. Resultados de la Velocidad de Pulso Ultrasónico. 
Para poder interpretar los resultados obtenidos partiremos de los criterios manejados por la RED DURAR (tabla 2) la cual establece que entre mayor sea el valor de resistividad eléctrica, mejor será el comportamiento del mortero. Una resistividad eléctrica alta nos indica que la porosidad del material es baja, lo cual es bueno ya que el mortero será resistente a afectaciones por agentes externos, como la corrosión. Los resultados obtenidos en la prueba de resistividad eléctrica se muestran en la figura \#4.

Tabla 2. Criterio General de valores de resistividad eléctrica del concreto. (CYTED-

DURAR, 1998)

\begin{tabular}{|c|c|c|}
\hline \multirow{2}{*}{$\begin{array}{l}\text { Riesgo a la } \\
\text { corrosión }\end{array}$} & \multicolumn{2}{|c|}{ 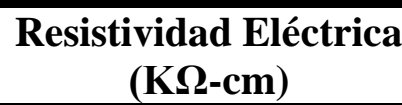 } \\
\hline & Desde & Hasta \\
\hline Alto riesgo & $<10$ & \\
\hline $\begin{array}{c}\text { Moderado } \\
\text { riesgo }\end{array}$ & 10 & 200 \\
\hline Poco riesgo & $>200$ & \\
\hline
\end{tabular}

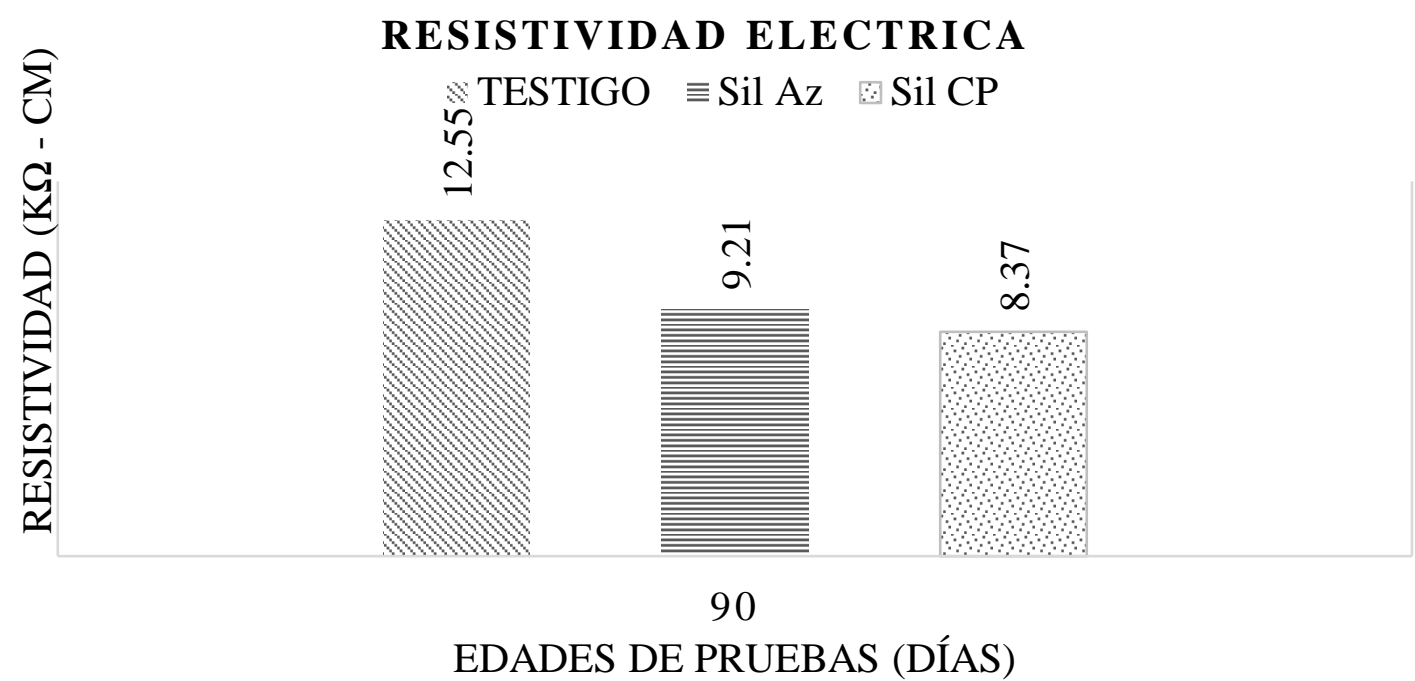

Figura 4. Resultados de Resistividad Eléctrica.

Uno de los ensayos que más nos proporciona información sobre las características del mortero es el de compresión simple, pues este ensayo nos establece parámetros generales sobre las propiedades y calidad del mortero. La prueba a compresión simple para el caso de morteros es la única que esta normada para especímenes cúbicos de $5 \mathrm{~cm}$ de arista.

A los 90 días la actividad puzolánica en la sustitución de sílice de los Azufres presenta un mejoramiento de $2.5 \%$ respecto a la muestra testigo (figura \#5). 


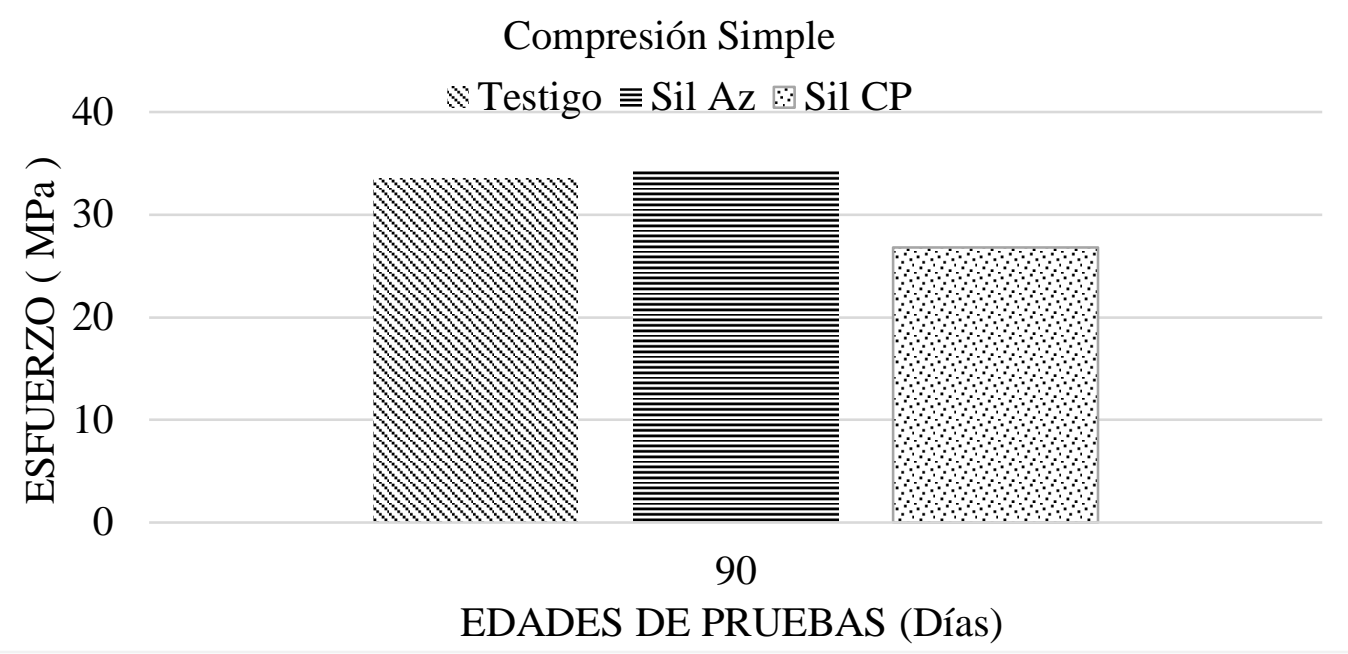

Figura 5. Resultados del ensayo a compresión simple

De acuerdo con la figura \#6 se observa que el ciclo cero no presenta perdida ni ganancia de masa, ya que durante este ciclo aún no se han expuesto las muestras a sulfato. A partir del ciclo 2 las sales comienzan a solidificarse dentro de la matriz provocando que ocurra ganancia de masa en las sustituciones y pérdida de esta para la muestra control.

La norma establece que la perdida permisible de masa es del $5 \%$, por lo cual se observa que a ambas edades las sustituciones presentan un buen comportamiento.

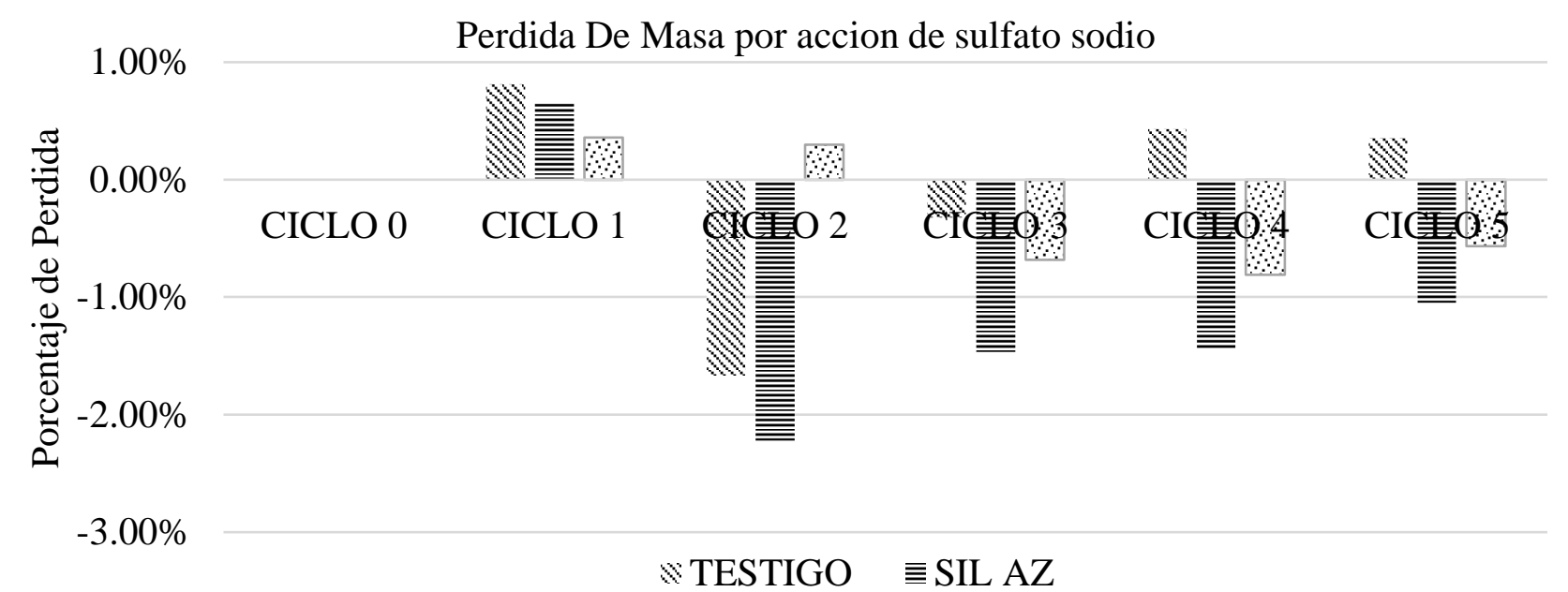

Figura 6. Resultados de pérdida de masa a 90 días.

La VPU durante el monitoreo de los ciclos de pérdida de masa presenta resultados muy estables. De acuerdo con criterios de la RED DURAR (tabla 1) la calidad de los morteros es ALTA. Se observa además que las sustituciones presentan un muy buen comportamiento con referencia a la muestra control. Figura \#7. 


\section{Velocidad De Pulso Ultrasónico}

\section{«TESTIGO $\equiv$ SIL AZ SIL CP}

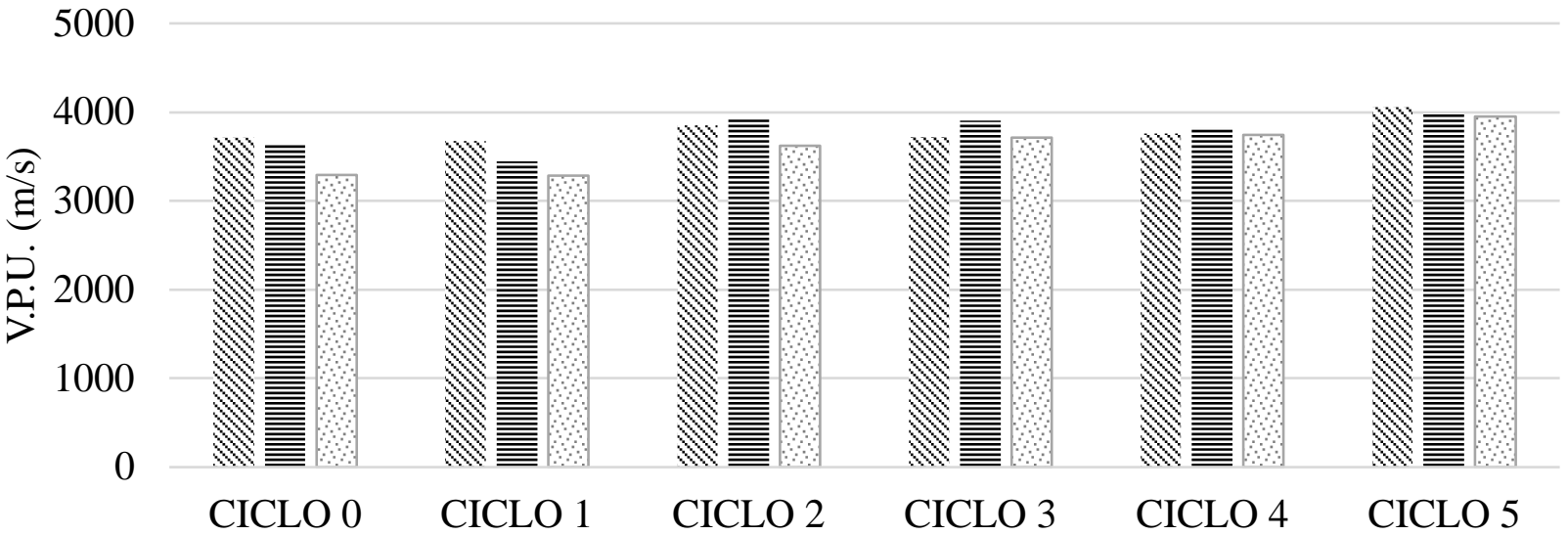

Figura 7. Resultados Velocidad de pulso ultrasónico a 90 días ante ataque por sulfatos.

Los resultados de la prueba de resistividad eléctrica durante los ciclos de pérdida de masa se presentan en la figura \#8. Se observa que los valores más altos de resistividad eléctrica y el comportamiento más estable ante ataque de sulfatos, lo presenta la mezcla Sil Az.

Por lo general las mezclas alcanzan los valores máximos durante el tercer ciclo, ya que a partir de este ciclo las sales comienzan a solidificarse dentro de la matriz.

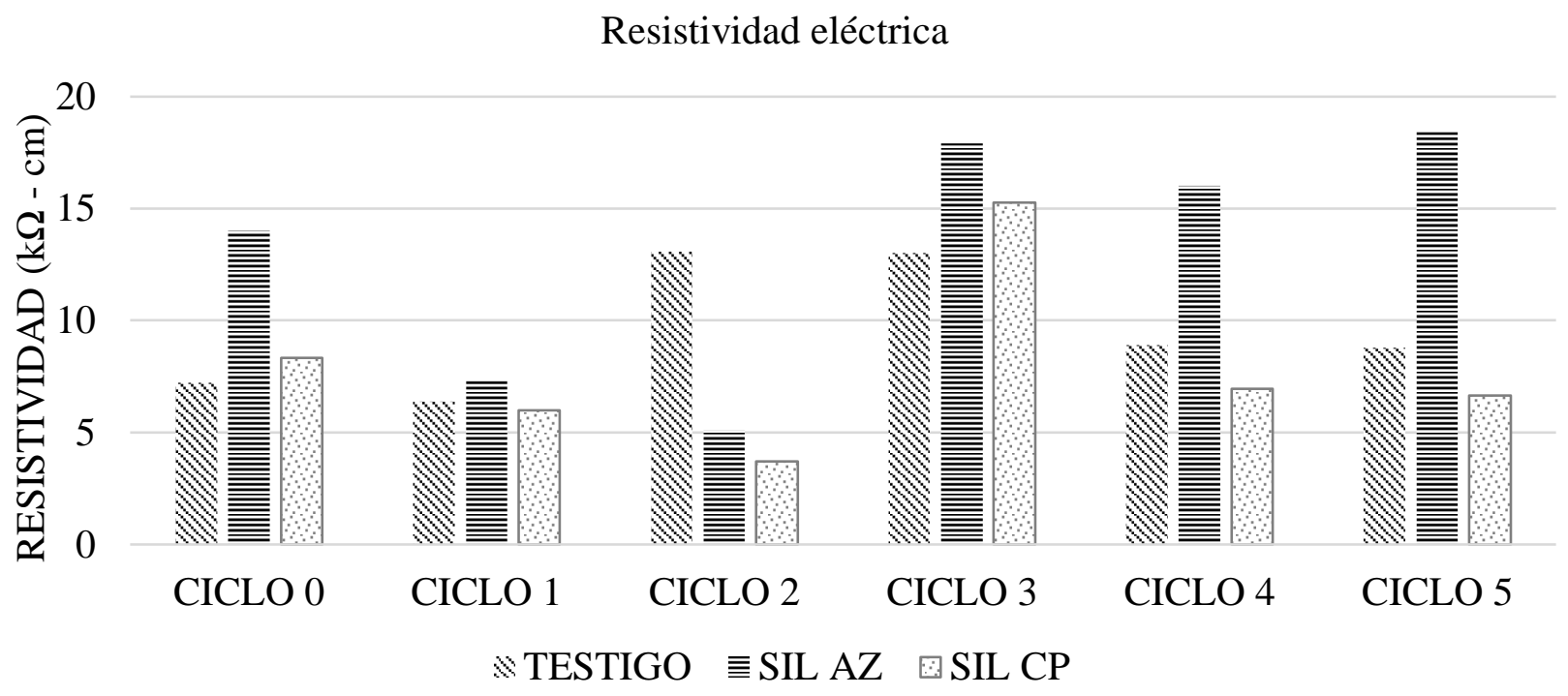

Figura 8. Resistividad eléctrica a los 90 días ante ataque por sulfatos.

\section{CONCLUSIONES}

De acuerdo con los resultados obtenidos, para una sustitución del 5\% de sílice, las dos mezclas de mortero con sustitución presentan los siguientes comportamientos:

La sustitución base CP por sílice (5\%) proveniente de la planta geotérmica Los Azufres, en la elaboración de morteros realizados para esta investigación, registra un comportamiento uniforme ante pruebas de evaluación mecánica y durabilidad. Comprueba ser un reemplazo que disminuye la cantidad de $\mathrm{CP}$ en las mezclas densificando la matriz y proporcionado un comportamiento mecánico superior. 
Además de que presenta mejor desempeño ante ensayos por durabilidad que los morteros convencionales a base de CP.

La sílice proveniente de Cerro Prieto no presenta valores presumiblemente favorables a compresión, sin embargo, logra mejorar la calidad del mortero.

Al elegir trabajar con un material puzolánica como la sílice proveniente de incrustaciones en las plantas geotérmicas, no sólo ayudamos a solucionar uno de los mayores problemas de esta industria, sino que a su vez también le estamos dando uso a un material proveniente de un proceso geotérmico. Por lo tanto, estamos colaborando con el medio ambiente en dos formas: reutilizando un geomaterial (del cual Michoacán y por supuesto México son una fuente potencial generadora de este subproducto) y disminuyendo la cantidad del uso de cemento portland en la construcción y por consecuencia las emisiones de $\mathrm{CO} 2$ al ambiente.

\section{AGRADECIMIENTOS}

Agradecimiento a todo el personal del Laboratorio de materiales “Ing. Luis Silva Ruelas” de la Facultad de Ingeniería Civil de la Universidad Michoacana de San Nicolás de Hidalgo por todo el soporte técnico, por las facilidades otorgadas en el uso de instalaciones y equipos de prueba.

\section{REFERENCIAS}

CYTED-DURAR. (1998). Durabilidad de la Armadura, Manual de Inspección, Evaluación y Diagnóstico de Corrosión en Estructuras de Hormigón Armado. Red Temática XV.

NMX-C-083-ONNCCE. (2014). Industria de la Construcción - Concreto - Determinación de la Resistencia de Cilindros de Concreto - Método de Prueba. Organismo Nacional de Normalización y Certificación de la Construcción y la Edificación, S.C.

NMX-C-514-ONNCCE-2016. (s.f.). Industria de la Construcción. Resistividad Eléctrica del Concreto Hidráulico. Especificaciones y Métodos de ensayo.

NMX-C-275-ONNCCE-2004. Industria de la Construcción-Concreto-Determinación de la Velocidad de Pulso a traves del Concreto-Método de Ultrasonido.

ASTM C 109M, I. Standard Test Method for Compressive Strength of Hydraulic Cement Mortars (Using 2-in. or [50-mm] Cube Specimens). (2002). U.S.A.

ASTM C 88-9a. Standard Test Method for Soundness of Aggregates by Use of Sodium Sulfate or Magnesium Sulfate.

BS-4027:1980. (2000). Especificación de Cemento Resistente a los Sulfatos. Inglaterra: British Standards Institution.

Navarro L. M., Martínez, W., Espinoza, J. A. (2011). Análisis de Materiales (Tercera edicion ed.). Morelia: Universidad Michoacana de San Nicolas de Hidalgo. 